\title{
Characterization of Structural Hemoglobin Variants by Top-Down Mass Spectrometry and R Programming Tools for Rapid Identification
}

\author{
Yuan Lin ${ }^{1}$, Archana M. Agarwal| ${ }^{3,4}$, Alan G. Marshall1 ${ }^{1,2^{*}}$, Lissa C Anderson²* \\ 1. Department of Chemistry and Biochemistry, Florida State University, Tallahassee, FL 32308 \\ 2. Ion Cyclotron Resonance Program, National High Magnetic Field Laboratory, Florida State University, \\ Tallahassee, FL 32310 \\ 3. Department of Pathology, University of Utah School of Medicine, Salt Lake City, UT 84132 \\ 4. ARUP Institute for Clinical and Experimental Pathology, Salt Lake City, UT 84108
}

Note: This work was performed at the National High Magnetic Field Laboratory lon Cyclotron Resonance User Facility, which is supported by the National Science Foundation Divisions of Chemistry and Materials Research through Cooperative Agreement No. DMR-1644779 and the State of Florida.

The R scripts are open source and freely available at the NHMFL ICR GitHub page (https://github.com/nhmfl-icr/VariantsID)

Raw data and various metadata can be accessed at the Open Science Framework DOI 10.17605/OSF.IO/ZPGYU

\section{Contents}

Table S1. Sequence coverage of $\mathrm{Hb}$ A obtained with chimeric ion loading MS2 spectra ....................... 1

Table S2. Diagnostic ion indices for substitution of each amino acid residue in the $\mathrm{Hb} A \beta$ subunit ........ 2

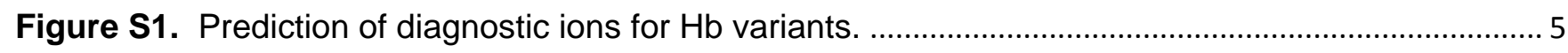

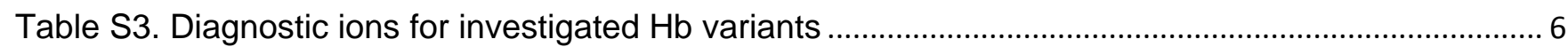

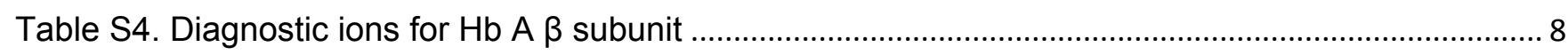

Figure S2. Hb Lepore-Washington-Boston heterozygous $\beta$ subunit MS1 spectrum and simulated spectrum. 
Table S1. Sequence coverage of Hb A obtained with chimeric ion loading MS2 spectra

(a) Sequence coverage for the $\mathrm{Hb} \mathrm{A}$ a subunit

\begin{tabular}{c|ccc}
\hline & & Charge State & \\
\hline CID/ETD iterations & $\mathbf{1 6 +}$ & $\mathbf{1 7 +}$ & $\mathbf{1 8 +}$ \\
$\mathbf{1 0 / 1 0}$ & $83 \%$ & $81 \%$ & $\mathbf{7 9 \%}$ \\
$\mathbf{8 / 1 2}$ & $82 \%$ & $\mathbf{7 9 \%}$ & $80 \%$ \\
$\mathbf{6 / 1 4}$ & $86 \%$ & $81 \%$ & $84 \%$ \\
$\mathbf{5 / 1 5}$ & $83 \%$ & $76 \%$ & $84 \%$ \\
$\mathbf{4 / 1 6}$ & $79 \%$ & $76 \%$ & $69 \%$ \\
$\mathbf{2 / 1 8}$ & $81 \%$ & $76 \%$ & $75 \%$ \\
\hline
\end{tabular}

(b) Sequence coverage for the $\mathrm{Hb} A \beta$ subunit.

\begin{tabular}{c|ccc}
\hline & \multicolumn{3}{c}{ Charge State } \\
\hline CID/ETD iterations & $\mathbf{1 6 +}$ & $\mathbf{1 7 +}$ & $\mathbf{1 8 +}$ \\
$\mathbf{1 0 / 1 0}$ & $71 \%$ & $74 \%$ & $70 \%$ \\
$\mathbf{8 / 1 2}$ & $75 \%$ & $79 \%$ & $76 \%$ \\
$\mathbf{6 / 1 4}$ & $74 \%$ & $76 \%$ & $68 \%$ \\
$\mathbf{5 / 1 5}$ & $70 \%$ & $77 \%$ & $72 \%$ \\
$\mathbf{4 / 1 6}$ & $74 \%$ & $76 \%$ & $68 \%$ \\
$\mathbf{2 / 1 8}$ & $64 \%$ & $70 \%$ & $68 \%$ \\
\hline
\end{tabular}


Table S2. Diagnostic ion indices for substitution of each amino acid residue in the $\mathrm{Hb} A \boldsymbol{\beta}$ subunit

\begin{tabular}{|c|c|c|c|c|c|}
\hline mut.site_N ${ }^{1}$ & WT.AA $^{2}$ & diag.N.term.start ${ }^{3}$ & diag.N.term.end ${ }^{4}$ & diag.C.term.start ${ }^{5}$ & diag.C.term.end $^{6}$ \\
\hline 1 & $\mathrm{~V}$ & 1 & 10 & 146 & 146 \\
\hline 2 & $\mathrm{H}$ & 2 & 62 & 145 & 146 \\
\hline 3 & L & 3 & 13 & 144 & 146 \\
\hline 4 & $\mathrm{~T}$ & 4 & 11 & 143 & 146 \\
\hline 5 & P & 5 & 35 & 142 & 146 \\
\hline 6 & $E$ & 6 & 21 & 141 & 146 \\
\hline 7 & $E$ & 7 & 21 & 140 & 146 \\
\hline 8 & K & 8 & 16 & 139 & 146 \\
\hline 9 & $S$ & 9 & 43 & 138 & 146 \\
\hline 10 & A & 10 & 12 & 137 & 146 \\
\hline 11 & V & 11 & 17 & 136 & 145 \\
\hline 12 & $\mathrm{~T}$ & 12 & 37 & 135 & 142 \\
\hline 13 & A & 13 & 26 & 134 & 136 \\
\hline 14 & L & 14 & 27 & 133 & 143 \\
\hline 15 & W & 15 & 36 & 132 & 146 \\
\hline 16 & G & 16 & 23 & 131 & 146 \\
\hline 17 & K & 17 & 58 & 130 & 138 \\
\hline 18 & V & 18 & 19 & 129 & 135 \\
\hline 19 & $\mathrm{~N}$ & 19 & 56 & 128 & 146 \\
\hline 20 & V & 20 & 22 & 127 & 128 \\
\hline 21 & D & 21 & 46 & 126 & 146 \\
\hline 22 & $E$ & 22 & 25 & 125 & 139 \\
\hline 23 & V & 23 & 32 & 124 & 126 \\
\hline 24 & $G$ & 24 & 28 & 123 & 130 \\
\hline 25 & G & 25 & 28 & 122 & 130 \\
\hline 26 & $E$ & 26 & 42 & 121 & 124 \\
\hline 27 & A & 27 & 52 & 120 & 133 \\
\hline 28 & L & 28 & 30 & 119 & 132 \\
\hline 29 & G & 29 & 45 & 118 & 121 \\
\hline 30 & $\mathrm{R}$ & 30 & 39 & 117 & 146 \\
\hline 31 & L & 31 & 47 & 116 & 118 \\
\hline 32 & L & 32 & 47 & 115 & 118 \\
\hline 33 & V & 33 & 53 & 114 & 123 \\
\hline 34 & V & 34 & 53 & 113 & 113 \\
\hline 35 & $Y$ & 35 & 129 & 112 & 146 \\
\hline 36 & $P$ & 36 & 50 & 111 & 141 \\
\hline 37 & W & 37 & 146 & 110 & 131 \\
\hline 38 & $\mathrm{~T}$ & 38 & 49 & 109 & 134 \\
\hline 39 & $Q$ & 39 & 126 & 108 & 146 \\
\hline 40 & $\mathrm{R}$ & 40 & 103 & 107 & 116 \\
\hline 41 & $F$ & 41 & 44 & 106 & 146 \\
\hline 42 & $F$ & 42 & 44 & 105 & 146 \\
\hline
\end{tabular}

${ }^{1}$ The amino acid (AA) position from $\mathrm{N}$-terminus

${ }^{2} \mathrm{AA}$ sequence for $\mathrm{HbA} \beta$ subunit

${ }^{3}$ The beginning number of theoretical diagnostic ion indices for each AA from the N-terminus

${ }^{4}$ The ending number of theoretical diagnostic ion indices for each AA from the N-terminus

${ }^{5}$ The beginning number of theoretical diagnostic ion indices for each $\mathrm{AA}$ from the $\mathrm{C}$-terminus

${ }^{6}$ The ending number of theoretical diagnostic ion indices for each AA from the C-terminus 


\begin{tabular}{|c|c|c|c|c|c|}
\hline 43 & $\mathrm{E}$ & 43 & 89 & 104 & 120 \\
\hline 44 & $S$ & 44 & 48 & 103 & 137 \\
\hline 45 & $\mathrm{~F}$ & 45 & 70 & 102 & 104 \\
\hline 46 & $G$ & 46 & 55 & 101 & 117 \\
\hline 47 & D & 47 & 51 & 100 & 125 \\
\hline 48 & $\mathrm{~L}$ & 48 & 67 & 99 & 114 \\
\hline 49 & $S$ & 49 & 71 & 98 & 102 \\
\hline 50 & $\mathrm{~T}$ & 50 & 83 & 97 & 108 \\
\hline 51 & $P$ & 51 & 57 & 96 & 110 \\
\hline 52 & D & 52 & 72 & 95 & 99 \\
\hline 53 & A & 53 & 61 & 94 & 119 \\
\hline 54 & $\mathrm{~V}$ & 54 & 59 & 93 & 112 \\
\hline 55 & $M$ & 55 & 146 & 92 & 146 \\
\hline 56 & $\mathrm{G}$ & 56 & 63 & 91 & 100 \\
\hline 57 & $\mathrm{~N}$ & 57 & 79 & 90 & 127 \\
\hline 58 & $\mathrm{P}$ & 58 & 99 & 89 & 95 \\
\hline 59 & $\mathrm{~K}$ & 59 & 60 & 88 & 129 \\
\hline 60 & $\mathrm{~V}$ & 60 & 66 & 87 & 92 \\
\hline 61 & $\mathrm{~K}$ & 61 & 64 & 86 & 87 \\
\hline 62 & A & 62 & 69 & 85 & 93 \\
\hline 63 & $\mathrm{H}$ & 63 & 76 & 84 & 144 \\
\hline 64 & $G$ & 64 & 68 & 83 & 90 \\
\hline 65 & $\mathrm{~K}$ & 65 & 81 & 82 & 85 \\
\hline 66 & $\mathrm{~K}$ & 66 & 81 & 81 & 85 \\
\hline 67 & V & 67 & 97 & 80 & 86 \\
\hline 68 & $\mathrm{~L}$ & 68 & 74 & 79 & 98 \\
\hline 69 & $\mathrm{G}$ & 69 & 73 & 78 & 82 \\
\hline 70 & $A$ & 70 & 75 & 77 & 84 \\
\hline 71 & $\mathrm{~F}$ & 71 & 84 & 76 & 101 \\
\hline 72 & $S$ & 72 & 88 & 75 & 97 \\
\hline 73 & D & 73 & 78 & 74 & 94 \\
\hline 74 & $\mathrm{G}$ & 74 & 82 & 73 & 77 \\
\hline 75 & $\mathrm{~L}$ & 75 & 77 & 72 & 78 \\
\hline 76 & $A$ & 76 & 85 & 71 & 76 \\
\hline 77 & $\mathrm{H}$ & 77 & 91 & 70 & 83 \\
\hline 78 & $\mathrm{~L}$ & 78 & 80 & 69 & 71 \\
\hline 79 & D & 79 & 93 & 68 & 73 \\
\hline 80 & $\mathrm{~N}$ & 80 & 101 & 67 & 89 \\
\hline 81 & $\mathrm{~L}$ & 81 & 87 & 66 & 68 \\
\hline 82 & $\mathrm{~K}$ & 82 & 94 & 65 & 80 \\
\hline 83 & G & 83 & 106 & 64 & 72 \\
\hline 84 & $\mathrm{~T}$ & 84 & 86 & 63 & 96 \\
\hline 85 & $\mathrm{~F}$ & 85 & 102 & 62 & 75 \\
\hline 86 & A & 86 & 114 & 61 & 70 \\
\hline 87 & $\mathrm{~T}$ & 87 & 122 & 60 & 62 \\
\hline 88 & $\mathrm{~L}$ & 88 & 90 & 59 & 65 \\
\hline 89 & $S$ & 89 & 146 & 58 & 74 \\
\hline 90 & $E$ & 90 & 100 & 57 & 103 \\
\hline 91 & $\mathrm{~L}$ & 91 & 95 & 56 & 58 \\
\hline 92 & $\mathrm{H}$ & 92 & 96 & 55 & 69 \\
\hline 93 & C & 93 & 111 & 54 & 146 \\
\hline 94 & $D$ & 94 & 98 & 53 & 67 \\
\hline 95 & $\mathrm{~K}$ & 95 & 119 & 52 & 64 \\
\hline 96 & $\mathrm{~L}$ & 96 & 104 & 51 & 55 \\
\hline 97 & $\mathrm{H}$ & 97 & 115 & 50 & 54 \\
\hline 98 & V & 98 & 108 & 49 & 79 \\
\hline 99 & D & 99 & 146 & 48 & 52 \\
\hline 100 & $P$ & 100 & 123 & 47 & 88 \\
\hline 101 & $E$ & 101 & 120 & 46 & 56 \\
\hline 102 & $\mathrm{~N}$ & 102 & 107 & 45 & 66 \\
\hline 103 & $F$ & 103 & 117 & 44 & 61 \\
\hline 104 & $\mathrm{R}$ & 104 & 146 & 43 & 106 \\
\hline
\end{tabular}




\begin{tabular}{|c|c|c|c|c|c|}
\hline 105 & $\mathrm{~L}$ & 105 & 109 & 42 & 50 \\
\hline 106 & $\mathrm{~L}$ & 106 & 109 & 41 & 50 \\
\hline 107 & $G$ & 107 & 118 & 40 & 63 \\
\hline 108 & $N$ & 108 & 138 & 39 & 44 \\
\hline 109 & V & 109 & 110 & 38 & 48 \\
\hline 110 & L & 110 & 113 & 37 & 40 \\
\hline 111 & V & 111 & 112 & 36 & 37 \\
\hline 112 & C & 112 & 146 & 35 & 53 \\
\hline 113 & V & 113 & 125 & 34 & 35 \\
\hline 114 & $\mathrm{~L}$ & 114 & 140 & 33 & 36 \\
\hline 115 & A & 115 & 127 & 32 & 60 \\
\hline 116 & $\mathrm{H}$ & 116 & 142 & 31 & 49 \\
\hline 117 & $\mathrm{H}$ & 117 & 142 & 30 & 49 \\
\hline 118 & $F$ & 118 & 121 & 29 & 43 \\
\hline 119 & $G$ & 119 & 135 & 28 & 39 \\
\hline 120 & K & 120 & 131 & 27 & 51 \\
\hline 121 & $E$ & 121 & 146 & 26 & 45 \\
\hline 122 & $F$ & 122 & 146 & 25 & 28 \\
\hline 123 & $\mathrm{~T}$ & 123 & 146 & 24 & 59 \\
\hline 124 & $P$ & 124 & 146 & 23 & 46 \\
\hline 125 & $P$ & 125 & 146 & 22 & 46 \\
\hline 126 & V & 126 & 132 & 21 & 33 \\
\hline 127 & Q & 127 & 130 & 20 & 107 \\
\hline 128 & A & 128 & 134 & 19 & 31 \\
\hline 129 & A & 129 & 134 & 18 & 31 \\
\hline 130 & $Y$ & 130 & 144 & 17 & 111 \\
\hline 131 & Q & 131 & 146 & 16 & 19 \\
\hline 132 & $\mathrm{~K}$ & 132 & 143 & 15 & 26 \\
\hline 133 & V & 133 & 136 & 14 & 20 \\
\hline 134 & V & 134 & 136 & 13 & 20 \\
\hline 135 & A & 135 & 137 & 12 & 17 \\
\hline 136 & $G$ & 136 & 146 & 11 & 27 \\
\hline 137 & V & 137 & 146 & 10 & 12 \\
\hline 138 & A & 138 & 139 & 9 & 11 \\
\hline 139 & $\mathrm{~N}$ & 139 & 146 & 8 & 38 \\
\hline 140 & A & 140 & 141 & 7 & 8 \\
\hline 141 & $\mathrm{~L}$ & 141 & 146 & 6 & 32 \\
\hline 142 & A & 142 & 146 & 5 & 6 \\
\hline 143 & $\mathrm{H}$ & 143 & 145 & 4 & 29 \\
\hline 144 & K & 144 & 146 & 3 & 14 \\
\hline 145 & $Y$ & 145 & 146 & 2 & 16 \\
\hline 146 & $\mathrm{H}$ & 146 & 146 & 1 & 3 \\
\hline
\end{tabular}


(a) Step 1-Compare $\beta$ variant sequence to sequence of $\mathrm{Hb} \mathrm{A} \beta$, obtain the mutation sites

Hb A $\beta$ VHLTPEEKSA.. HbS $\beta$ VHLTPVEKSA...

\begin{tabular}{ccccc} 
Variants & $\begin{array}{c}\text { mut. } \\
\text { site_N }\end{array}$ & $\begin{array}{c}\text { WT. } \\
\text { AA }\end{array}$ & $\begin{array}{c}\text { MT. } \\
\text { AA }\end{array}$ & $\begin{array}{c}\Delta \text { mass } \\
\text { (Da) }\end{array}$ \\
\hline Hb S & 6 & E & V & -29.9742
\end{tabular}

(b) Step 2-Use Table S2 to determine type and index of potential diagnostic ions

\begin{tabular}{ccccc}
\hline \multicolumn{4}{c}{ diag.N. diag.N. diag.C. diag.C. } \\
Variants & $\begin{array}{c}\text { term. } \\
\text { start }\end{array}$ & $\begin{array}{c}\text { term. } \\
\text { end }\end{array}$ & $\begin{array}{c}\text { term. } \\
\text { start }\end{array}$ & $\begin{array}{c}\text { term. } \\
\text { end }\end{array}$ \\
\hline Hb S & 6 & 21 & 141 & 146 \\
\hline
\end{tabular}

(c) Step 3-Identify diagnostic ions from step 2 that match the ion type and index of $\mathrm{Hb} \mathrm{A} \beta$ product ions (Table S4) in experimental MS2 data

\begin{tabular}{cccccc}
\hline Ion & Mass (Da) & Variant & Mutation & $\Delta$ mass (Da) \\
\hline b7 & 776.4301 & Hb S & Glu6Val & -29.9742 \\
b8 & 904.5251 & Hb S & Glu6Val & -29.9742 \\
b10 & 1062.594 & Hb S & Glu6Val & -29.9742 \\
b11 & 1161.663 & Hb S & Glu6Val & -29.9742 \\
b14 & 1446.831 & Hb S & Glu6Val & -29.9742 \\
b20 & 2130.207 & Hb S & Glu6Val & -29.9742 \\
c7 & 793.4567 & Hb S & Glu6Val & -29.9742 \\
c8 & 921.5516 & Hb S & Glu6Val & -29.9742 \\
c9 & 1008.584 & Hb S & Glu6Val & -29.9742 \\
c11 & 1178.689 & Hb S & Glu6Val & -29.9742 \\
c12 & 1279.737 & Hb S & Glu6Val & -29.9742 \\
c13 & 1350.774 & Hb S & Glu6Val & -29.9742 \\
c14 & 1463.858 & Hb S & Glu6Val & -29.9742 \\
c15 & 1649.937 & Hb S & Glu6Val & -29.9742 \\
\hline
\end{tabular}

Figure S1. Prediction of diagnostic ions for $\mathrm{Hb}$ variants. (a) Comparison of $\mathrm{Hb}$ variants sequences and $\mathrm{Hb} A$ subunit, $\mathrm{Hb}$ variants sequences contains the $\mathrm{N}$-terminal methionine and are in one FASTA file. (b) Table $\mathbf{S 2}$ is used to determine type and index of potential diagnostic ions, for $\mathrm{Hb} \mathrm{S}$ the potential diagnostic ions are b-/c- ions with residue index between 6 to 21, and $\mathrm{y}$-/z•- ions with residue index between 141 to 146. (c) The predicted diagnostic ions for $\mathrm{Hb} \mathrm{S}$ referring to $\mathrm{Hb} \mathrm{A} \beta$ reference base (Table S4). For each $\mathrm{Hb}$ variant, only two diagnostic product ions are listed for illustration purposes. 
Table S3. Diagnostic ions for investigated $\mathrm{Hb}$ variants

\begin{tabular}{|c|c|c|c|c|c|c|}
\hline Diagnostic ions & Ion.type & Ion.num & Mass (Da) & Variant & Mutation & Delta mass \\
\hline y8 & $y$ & 8 & 972.5374 & Hb Abruzzo & His143Arg & 19.0422 \\
\hline y11 & $y$ & 11 & 1199.6644 & Hb Abruzzo & His143Arg & 19.0422 \\
\hline y12 & $y$ & 12 & 1270.7015 & Hb Abruzzo & His143Arg & 19.0422 \\
\hline y14 & $y$ & 14 & 1468.8383 & Hb Abruzzo & His143Arg & 19.0422 \\
\hline y22 & $y$ & 22 & 2354.3092 & Hb Abruzzo & His143Arg & 19.0422 \\
\hline y23 & $y$ & 23 & 2451.3619 & Hb Abruzzo & His143Arg & 19.0422 \\
\hline y24 & $y$ & 24 & 2552.4096 & Hb Abruzzo & His143Arg & 19.0422 \\
\hline y29 & $y$ & 29 & 3160.7054 & Hb Abruzzo & His143Arg & 19.0422 \\
\hline$z 11$ & z & 11 & 1183.6456 & Hb Abruzzo & His143Arg & 19.0422 \\
\hline z18 & z & 18 & 1943.0736 & Hb Abruzzo & His143Arg & 19.0422 \\
\hline z19 & z & 19 & 2014.1107 & Hb Abruzzo & His143Arg & 19.0422 \\
\hline z24 & $z$ & 24 & 2536.3909 & Hb Abruzzo & His143Arg & 19.0422 \\
\hline$z 26$ & z & 26 & 2812.5019 & Hb Abruzzo & His143Arg & 19.0422 \\
\hline z27 & $z$ & 27 & 2940.5968 & Hb Abruzzo & His143Arg & 19.0422 \\
\hline z29 & $z$ & 29 & 3144.6867 & Hb Abruzzo & His143Arg & 19.0422 \\
\hline b14 & $b$ & 14 & 1475.8580 & $\mathrm{Hb} \mathrm{C}$ & Glu6Lys & -0.9476 \\
\hline b20 & $b$ & 20 & 2159.2335 & $\mathrm{Hb} \mathrm{C}$ & Glu6Lys & -0.9476 \\
\hline c6 & $\mathrm{C}$ & 6 & 693.4406 & $\mathrm{Hb} \mathrm{C}$ & Glu6Lys & -0.9476 \\
\hline c7 & $\mathrm{C}$ & 7 & 822.4832 & $\mathrm{Hb} \mathrm{C}$ & Glu6Lys & -0.9476 \\
\hline c8 & $\mathrm{C}$ & 8 & 950.5782 & $\mathrm{Hb} \mathrm{C}$ & Glu6Lys & -0.9476 \\
\hline y35 & $y$ & 35 & 3800.9958 & $\mathrm{Hb} D$ & Glu121Gln & -0.9840 \\
\hline $\mathrm{z} 26$ & $z$ & 26 & 2792.4757 & $\mathrm{Hb} D$ & Glu121Gln & -0.9840 \\
\hline z28 & $z$ & 28 & 2977.5921 & $\mathrm{Hb} D$ & Glu121Gln & -0.9840 \\
\hline z29 & $z$ & 29 & 3124.6605 & $\mathrm{Hb} D$ & Glu121Gln & -0.9840 \\
\hline z30 & $\mathrm{z}$ & 30 & 3261.7194 & $\mathrm{Hb} D$ & Glu121Gln & -0.9840 \\
\hline $\mathbf{z} 40$ & $z$ & 40 & 4267.2624 & $\mathrm{Hb} D$ & Glu121Gln & -0.9840 \\
\hline b26 & $b$ & 26 & 2745.4570 & $\mathrm{Hb} \mathrm{E}$ & Glu26Lys & -0.9476 \\
\hline c26 & $\mathrm{C}$ & 26 & 2762.4836 & $\mathrm{Hb} \mathrm{E}$ & Glu26Lys & -0.9476 \\
\hline c29 & $\mathrm{c}$ & 29 & 3003.6262 & $\mathrm{Hb} \mathrm{E}$ & Glu26Lys & -0.9476 \\
\hline c39 & $\mathrm{C}$ & 39 & 4259.3339 & $\mathrm{Hb} \mathrm{E}$ & Glu26Lys & -0.9476 \\
\hline c40 & c & 40 & 4415.4350 & $\mathrm{Hb} \mathrm{E}$ & Glu26Lys & -0.9476 \\
\hline y28 & $y$ & 28 & 2980.5428 & Hb Riyadh & Lys120Asn & -14.0520 \\
\hline y29 & $y$ & 29 & 3127.6112 & Hb Riyadh & Lys120Asn & -14.0520 \\
\hline y31 & $y$ & 31 & 3401.7290 & Hb Riyadh & Lys120Asn & -14.0520 \\
\hline y33 & $y$ & 33 & 3585.8502 & Hb Riyadh & Lys120Asn & -14.0520 \\
\hline y36 & $y$ & 36 & 3886.9962 & Hb Riyadh & Lys120Asn & -14.0520 \\
\hline y47 & $y$ & 47 & 5139.6890 & Hb Riyadh & Lys120Asn & -14.0520 \\
\hline $\mathrm{z} 27$ & z & 27 & 2907.5026 & Hb Riyadh & Lys120Asn & -14.0520 \\
\hline z29 & $z$ & 29 & 3111.5925 & Hb Riyadh & Lys120Asn & -14.0520 \\
\hline z30 & $z$ & 30 & 3248.6514 & Hb Riyadh & Lys120Asn & -14.0520 \\
\hline z31 & z & 31 & 3385.7103 & Hb Riyadh & Lys120Asn & -14.0520 \\
\hline z32 & $z$ & 32 & 3456.7474 & Hb Riyadh & Lys120Asn & -14.0520 \\
\hline z39 & z & 39 & 4197.1729 & Hb Riyadh & Lys120Asn & -14.0520 \\
\hline $\mathrm{z} 43$ & $z$ & 43 & 4636.4636 & Hb Riyadh & Lys120Asn & -14.0520 \\
\hline z45 & $z$ & 45 & 4897.5750 & Hb Riyadh & Lys120Asn & -14.0520 \\
\hline $\mathrm{z} 46$ & $z$ & 46 & 5026.6175 & Hb Riyadh & Lys120Asn & -14.0520 \\
\hline z49 & $z$ & 49 & 5337.7657 & Hb Riyadh & Lys120Asn & -14.0520 \\
\hline z50 & $z$ & 50 & 5474.8246 & Hb Riyadh & Lys120Asn & -14.0520 \\
\hline b7 & $b$ & 7 & 776.4301 & $\mathrm{Hb} \mathrm{S}$ & Glu6Val & -29.9742 \\
\hline b8 & $b$ & 8 & 904.5251 & $\mathrm{Hb} \mathrm{S}$ & Glu6Val & -29.9742 \\
\hline b14 & $b$ & 14 & 1446.8315 & $\mathrm{Hb} \mathrm{S}$ & Glu6Val & -29.9742 \\
\hline b15 & $b$ & 15 & 1632.9108 & $\mathrm{Hb} \mathrm{S}$ & Glu6Val & -29.9742 \\
\hline b18 & $b$ & 18 & 1917.0956 & $\mathrm{Hb} \mathrm{S}$ & Glu6Val & -29.9742 \\
\hline b19 & $b$ & 19 & 2031.1386 & $\mathrm{Hb} \mathrm{S}$ & Glu6Val & -29.9742 \\
\hline b20 & $b$ & 20 & 2130.2070 & $\mathrm{Hb} \mathrm{S}$ & Glu6Val & -29.9742 \\
\hline b21 & $b$ & 21 & 2245.2339 & $\mathrm{Hb} \mathrm{S}$ & Glu6Val & -29.9742 \\
\hline c7 & $\mathrm{C}$ & 7 & 793.4567 & $\mathrm{Hb} \mathrm{S}$ & Glu6Val & -29.9742 \\
\hline c8 & $\mathrm{C}$ & 8 & 921.5516 & $\mathrm{Hb} \mathrm{S}$ & Glu6Val & -29.9742 \\
\hline c9 & C & 9 & 1008.5837 & $\mathrm{Hb} \mathrm{S}$ & Glu6Val & -29.9742 \\
\hline
\end{tabular}




\begin{tabular}{lllllll}
\hline $\mathbf{c 1 1}$ & $\mathrm{c}$ & 11 & 1178.6892 & $\mathrm{Hb} \mathrm{S}$ & Glu6Val & -29.9742 \\
$\mathbf{c 1 2}$ & $\mathrm{c}$ & 12 & 1279.7369 & $\mathrm{Hb} \mathrm{S}$ & Glu6Val & -29.9742 \\
$\mathbf{c 1 3}$ & $\mathrm{c}$ & 13 & 1350.7740 & $\mathrm{Hb} \mathrm{S}$ & Glu6Val & -29.9742 \\
$\mathbf{c 1 4}$ & $\mathrm{C}$ & 14 & 1463.8580 & $\mathrm{Hb} \mathrm{S}$ & Glu6Val & -29.9742 \\
$\mathbf{c 1 5}$ & $\mathrm{C}$ & 15 & 1649.9374 & $\mathrm{Hb} \mathrm{S}$ & Glu6Val & -29.9742 \\
$\mathbf{c 2 0}$ & $\mathrm{C}$ & 20 & 2147.2335 & $\mathrm{Hb} \mathrm{S}$ & Glu6Val & -29.9742 \\
$\mathbf{c 2 1}$ & $\mathrm{c}$ & 21 & 2262.2605 & $\mathrm{Hb} \mathrm{S}$ & Glu6Val & -29.9742 \\
\hline
\end{tabular}


Table S4. Diagnostic ions for $\mathrm{Hb} A \boldsymbol{\beta}$ subunit

\begin{tabular}{|c|c|c|c|c|c|c|c|}
\hline & & & & & & & \\
\hline Diagnostic ions & Ion.typ & Ion.nu & Mass (Da) & y11 & $\mathrm{y}$ & 11 & 1180.6222 \\
\hline b7 & b & 7 & 806.4043 & y13 & $y$ & 13 & 1350.7277 \\
\hline b8 & $\mathrm{b}$ & 8 & 934.4993 & y14 & $\mathrm{y}$ & 14 & 1449.7961 \\
\hline b11 & $\mathrm{b}$ & 11 & 1191.6368 & y15 & $\mathrm{y}$ & 15 & 1577.8911 \\
\hline b14 & $\mathrm{b}$ & 14 & 1476.8057 & y22 & $\mathrm{y}$ & 22 & 2335.2670 \\
\hline b20 & $b$ & 20 & 2160.1812 & y23 & $y$ & 23 & 2432.3197 \\
\hline b22 & $b$ & 22 & 2404.2507 & y24 & $\mathrm{y}$ & 24 & 2533.3674 \\
\hline b23 & $b$ & 23 & 2503.3191 & y29 & $\mathrm{y}$ & 29 & 3141.6633 \\
\hline b24 & $b$ & 24 & 2560.3406 & y30 & $y$ & 30 & 3278.7222 \\
\hline b27 & $b$ & 27 & 2817.4417 & y31 & $\mathrm{y}$ & 31 & 3415.7811 \\
\hline b30 & $b$ & 30 & 3143.6484 & y32 & $\mathrm{y}$ & 32 & 3486.8182 \\
\hline b31 & $b$ & 31 & 3256.7325 & y36 & $y$ & 36 & 3901.0483 \\
\hline b32 & $\mathrm{b}$ & 32 & 3369.8165 & y47 & $y$ & 47 & 5153.7411 \\
\hline b33 & $b$ & 33 & 3468.8849 & y48 & $y$ & 48 & 5268.7680 \\
\hline b34 & $b$ & 34 & 3567.9533 & y49 & $y$ & 49 & 5367.8364 \\
\hline b35 & $\mathrm{b}$ & 35 & 3731.0167 & y52 & $\mathrm{y}$ & 52 & 5746.0744 \\
\hline b40 & $\mathrm{b}$ & 40 & 4399.3561 & y53 & $\mathrm{y}$ & 53 & 5861.1013 \\
\hline b41 & $\mathrm{b}$ & 41 & 4546.4245 & y54 & $\mathrm{y}$ & 54 & 5964.1105 \\
\hline b42 & $b$ & 42 & 4693.4929 & y56 & $y$ & 56 & 6214.2535 \\
\hline b43 & $\mathrm{b}$ & 43 & 4822.5355 & y58 & $\mathrm{y}$ & 58 & 6430.3281 \\
\hline b45 & $b$ & 45 & 5056.6360 & y60 & $y$ & 60 & 6644.4598 \\
\hline b46 & $b$ & 46 & 5113.6574 & y64 & $y$ & 64 & 7020.6345 \\
\hline b47 & $b$ & 47 & 5228.6844 & y81 & $y$ & 81 & 8799.6024 \\
\hline b48 & $b$ & 48 & 5341.7685 & y92 & $y$ & 92 & 9947.2309 \\
\hline b49 & $b$ & 49 & 5428.8005 & y97 & $\mathrm{y}$ & 97 & 10430.4638 \\
\hline c5 & C & 5 & 565.3457 & y98 & $\mathrm{y}$ & 98 & 10517.4958 \\
\hline c7 & C & 7 & 823.4308 & y99 & $y$ & 99 & 10630.5799 \\
\hline c8 & C & 8 & 951.5258 & y100 & $y$ & 100 & 10745.6068 \\
\hline c9 & C & 9 & 1038.5578 & y101 & $\mathrm{y}$ & 101 & 10802.6283 \\
\hline c11 & c & 11 & 1208.6634 & y102 & $y$ & 102 & 10949.6967 \\
\hline c12 & C & 12 & 1309.7110 & y112 & $\mathrm{y}$ & 112 & 12291.3109 \\
\hline c13 & C & 13 & 1380.7482 & y118 & $\mathrm{y}$ & 118 & 12928.7384 \\
\hline c14 & C & 14 & 1493.8322 & y123 & $\mathrm{y}$ & 123 & 13355.9451 \\
\hline c15 & $\mathrm{C}$ & 15 & 1679.9115 & $z 14$ & $z$ & 14 & 1433.7774 \\
\hline c22 & C & 22 & 2421.2773 & z15 & z & 15 & 1561.8723 \\
\hline c33 & C & 33 & 3485.9115 & $z 17$ & z & 17 & 1852.9943 \\
\hline c34 & C & 34 & 3584.9799 & z18 & z & 18 & 1924.0314 \\
\hline c36 & C & 36 & 3845.0960 & z19 & $z$ & 19 & 1995.0685 \\
\hline c39 & C & 39 & 4260.2816 & z24 & z & 24 & 2517.3487 \\
\hline c40 & C & 40 & 4416.3827 & $z 25$ & z & 25 & 2664.4171 \\
\hline c42 & C & 42 & 4710.5195 & z26 & $z$ & 26 & 2793.4597 \\
\hline c43 & C & 43 & 4839.5621 & $z 27$ & z & 27 & 2921.5546 \\
\hline c44 & C & 44 & 4926.5941 & z28 & $z$ & 28 & 2978.5761 \\
\hline c54 & c & 54 & 5929.0599 & z29 & z & 29 & 3125.6445 \\
\hline c56 & C & 56 & 6117.1219 & z33 & z & 33 & 3583.8835 \\
\hline c59 & C & 59 & 6456.3125 & z39 & $z$ & 39 & 4211.2249 \\
\hline c60 & c & 60 & 6555.3810 & z40 & z & 40 & 4268.2464 \\
\hline c61 & $\mathrm{C}$ & 61 & 6683.4759 & z45 & z & 45 & 4911.6270 \\
\hline c62 & c & 62 & 6754.5130 & z48 & z & 48 & 5252.7493 \\
\hline c63 & C & 63 & 6891.5719 & z49 & z & 49 & 5351.8177 \\
\hline c64 & c & 64 & 6948.5934 & $\mathrm{z} 51$ & $z$ & 51 & 5601.9607 \\
\hline c69 & C & 69 & 7473.9573 & z52 & $z$ & 52 & 5730.0556 \\
\hline c72 & c & 72 & 7779.0948 & z53 & $\mathrm{z}$ & 53 & 5845.0826 \\
\hline c73 & C & 73 & 7894.1218 & z58 & z & 58 & 6414.3094 \\
\hline c80 & c & 80 & 8614.4773 & $z 61$ & z & 61 & 6699.4782 \\
\hline c101 & C & 101 & 10948.6451 & z64 & z & 64 & 7004.6158 \\
\hline c111 & c & 111 & 12074.3109 & z65 & z & 65 & 7132.7107 \\
\hline y6 & $y$ & 6 & 768.4151 & z66 & z & 66 & 7245.7948 \\
\hline y8 & $y$ & 8 & 953.4952 & z67 & z & 67 & 7359.8377 \\
\hline y9 & $y$ & 9 & 1024.5323 & $\mathrm{z} 68$ & z & 68 & 7474.8647 \\
\hline
\end{tabular}




\begin{tabular}{cccc}
\hline $\mathbf{z 6 9}$ & z & 69 & 7587.9487 \\
$\mathbf{z 7 3}$ & z & 73 & 7966.1503 \\
$\mathbf{z 7 4}$ & z & 74 & 8081.1772 \\
$\mathbf{z 7 7}$ & z & 77 & 8386.3148 \\
$\mathbf{z 7 9}$ & z & 79 & 8556.4203 \\
$\mathbf{z 8 1}$ & $\mathbf{z}$ & 81 & 8783.5837 \\
$\mathbf{z 8 2}$ & $\mathbf{z}$ & 82 & 8911.6787 \\
$\mathbf{z 8 3}$ & $\mathbf{z}$ & 83 & 8968.7001 \\
$\mathbf{z 8 5}$ & $\mathbf{z}$ & 85 & 9176.7962 \\
$\mathbf{z 8 6}$ & $\mathbf{z}$ & 86 & 9304.8911 \\
$\mathbf{z 1 2 4}$ & $\mathbf{z}$ & 124 & 13438.9948 \\
$\mathbf{z 1 3 8}$ & $\mathbf{z}$ & 138 & 14908.7463 \\
\hline
\end{tabular}


(a)

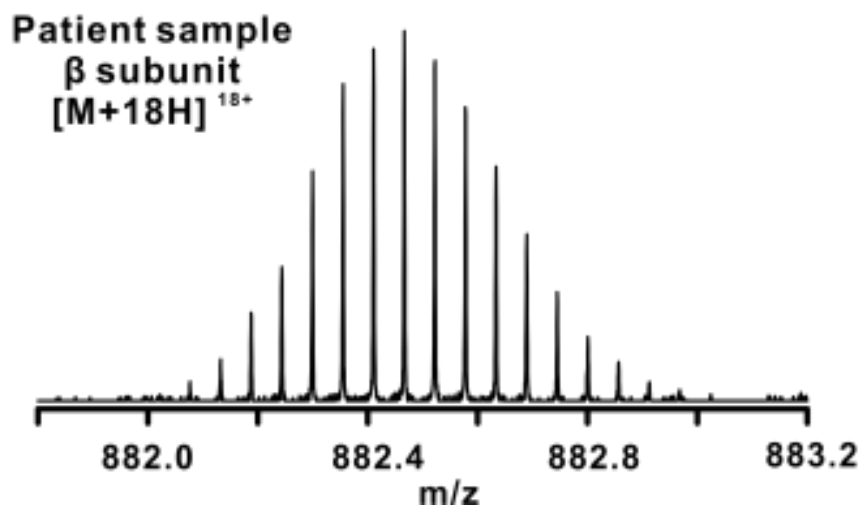

(b)

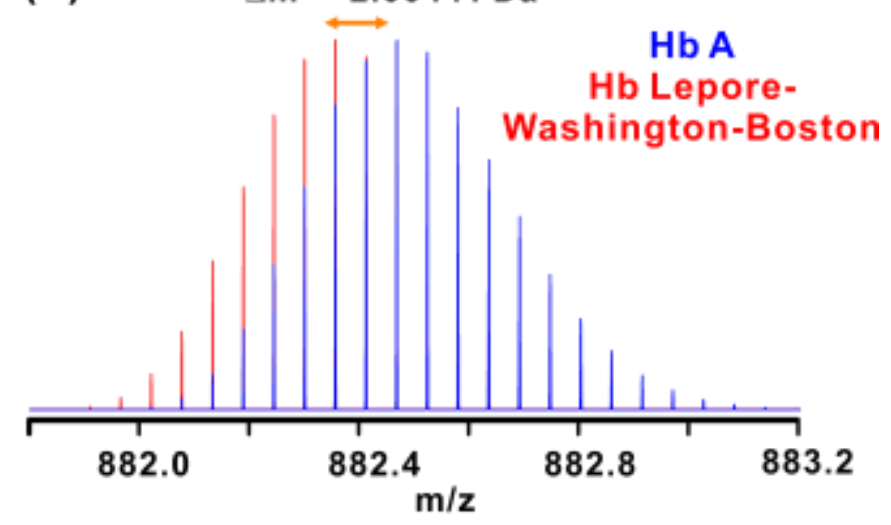

Figure S2. Hb Lepore-Washington-Boston heterozygous $\beta$ subunit MS1 spectrum and simulated spectrum. (a) Isotope distribution of $\mathrm{Hb}$ Lepore-Washington-Boston heterozygous $\beta$ subunit $18+$ charge state with a mass resolving power $(\mathrm{m} / \Delta \mathrm{m})$ of 400k. (b) Simulated isotope distribution of these two $\beta$ subunits $18+$ charge state with a mass resolving power $(\mathrm{m} / \Delta \mathrm{m})$ of 1.2 million. 